\title{
Ophthalmoplegic migraine and aberrant regeneration of the oculomotor nerve
}

\author{
JUSTIN O'DAY, FRANK BILLSON, AND JOHN KING \\ From the Department of Ophthalmology, University of Sydney, Sydney Eye Hospital, \\ and Department of Neurology, Royal Melbolirne Hospital, Australia
}

SUMMARY A patient with ophthalmoplegic migraine developed aberrant regeneration of the oculomotor nerve. This finding supports the view that the oculomotor nerve lesion in ophthalmoplegic migraine is peripheral, but its rarity suggests that the underlying mechanism may be ischaemic rather than compression by an oedematous intracavernous internal carotid artery.

Ophthalmoplegic migraine was first described by Charcot in $1890 . .^{1}$ In a typical case there is a long history of recurrent attacks of unilateral headache over many years prior to the headache being associated with ophthalmoplegia. The pain is usually more severe and protracted than in other forms of migraine, and after several days an ipsilateral oculomotor paralysis develops which may last from hours to many weeks. Less commonly the trochlear and abducens nerves have been involved. The attacks of extraocular muscle weakness become more severe with each attack until a permanent unilateral oculomotor palsy develops. ${ }^{2}$

The site of the lesion of the oculomotor nerve in ophthalmoplegic migraine was regarded as being in the cavernous sinus by Walsh and O'Doherty after the observation of a constricted intracavernous internal carotid artery on angiography in 2 patients. The mechanism whereby cranial palsies occurred was postulated to be a compression of the nerve by the oedematous carotid arterial wall.

Aberrant regeneration of the oculomotor nerve is seen during the recovery phase from acute palsies produced by aneurysms and head injury and rarely by tumours. In children it is common sequel to a congenital third nerve palsy. ${ }^{4}$ The same disorder has been reported in the absence of an acute oculomotor palsy in patients who were subsequently shown to have meningiomas of the cavernous sinus. ${ }^{5}$ We report the case of a patient with ophthalmoplegic migraine who developed aberrant regeneration of the oculomotor nerve.

\section{Case report}

A 45-year-old man was in good health until 1970,

Correspondence to Dr John King, Royal Melbourne Hospital, Victoria 3050, Australia. when he began to suffer from recurrent episodes of severe headaches associated with ophthalmoplegia. The headaches occurred at about 6-monthly intervals and were throbbing in nature. They began gradually over the right orbit and radiated back into the right occiput. They were associated with nausea and vomiting and resulted in his admission to hospital on at least 4 occasions. Typically the headaches would gradually increase in intensity over 4 days and then quickly subside after the onset of double vision. There was no previous history of headaches suggestive of migraine, nor family history of migraine. He sustained 8 episodes of complete right third nerve palsy, 2 of which were associated with fourth and sixth nerve palsies. The ocular palsy always involved the right eye, lasted 2 to 6 weeks, and was followed by a complete recovery of function.

In December 1976 painful ophthalmoplegia developed with involvement of the right third nerve. Six weeks later, when the patient's eyelids began to open, he noted blurred vision for near. On examination at that time he displayed typical features of a right third nerve paresis with aberrant regeneration. A right partial ptosis was present in the primary position with eye lid retraction in downgaze (Figs 1, 2, 3). There was a limitation of all movements of his right eye except abduction and intorsion. On attempted adduction and depression of the right eye, with distance fixation his right pupil constricted. In the primary gaze position his right pupil was mid-dilated and unresponsive to light. The general examination was normal and there were no other neurological abnormalities.

Skull $x$-rays and right carotid arteriography were normal. Cerebrospinal fluid examination, including VDRL, full blood examination, fasting blood sugar, and erythrocyte sedimentation rate were all normal. 


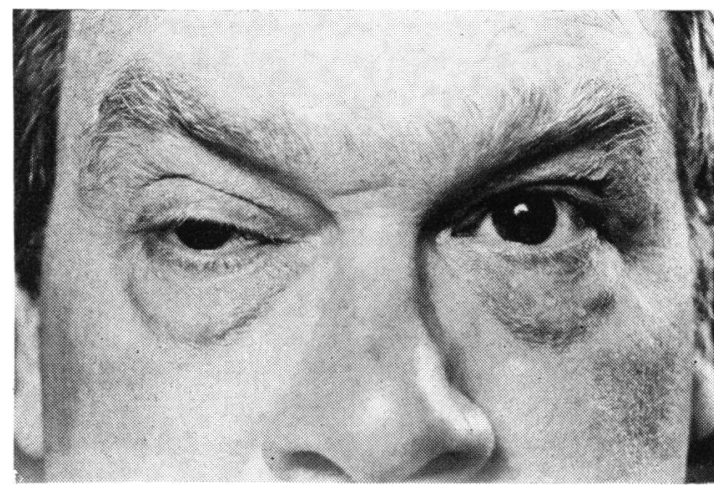

Fig. 1 Right partial ptosis in the primary position.

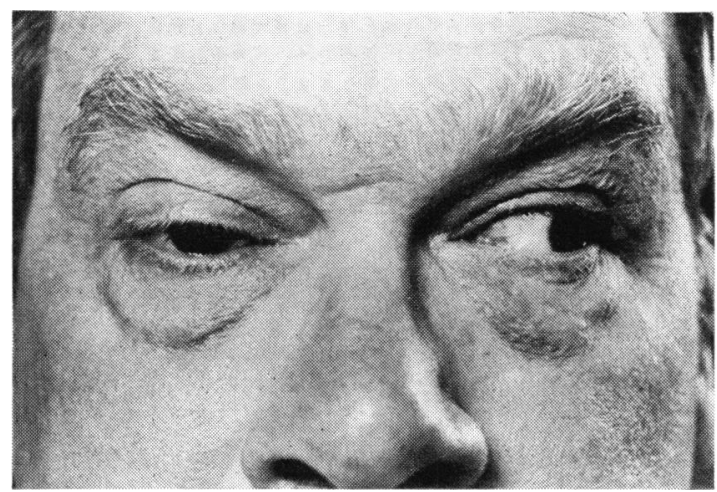

Fig. 3 Failure of adduction of the right eye on gaze to the left.

The clinical features of this patient are typical of ophthalmoplegic migraine apart from the absence of a history of migraine before the onset of the attacks of ophthalmoplegia. The painful ophthalmoplegia seen in diabetes mellitus was ruled out by blood sugar estimations, and carotid angiography excluded the presence of an aneurysm of the internal carotid artery or posterior communication artery. There was no evidence of neurosyphilis or other local infections.

\section{Discussion}

An important cause of recurrent painful ophthalmoplegia is the Tolosa-Hunt syndrome, ${ }^{6}$ which must be included in the differential diagnosis of ophthalmoplegic migraine. In this condition the pain, which usually lasts more than several days, may precede the ophthalmoplegia, and is described as a steady boring pain rather than a throbbing hemicrania. The neurological involvement is usually more extensive than in ophthalmoplegic migraine and has included involvement of the optic, oculo-

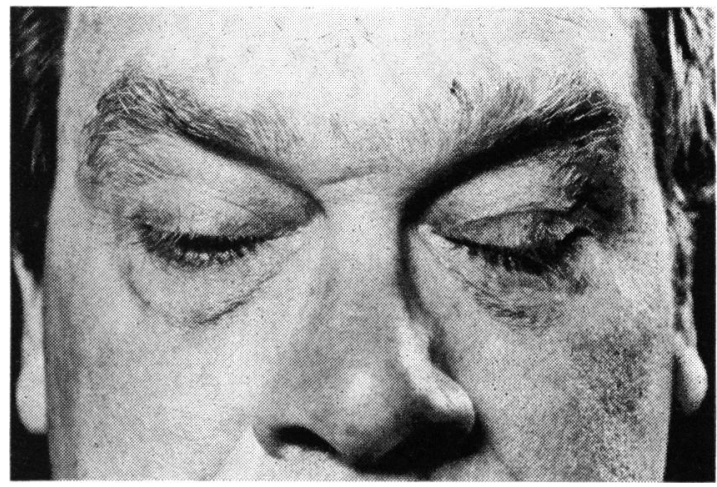

Fig. 2 Retraction of right upper lid on downward gaze.

motor, trochlear, ophthalmic division of the trigeminal, abducens, and ocular sympathetic nerves. In addition there may be proptosis greater than that which would be simply attributed to an oculomotor palsy. The condition is recurrent, with spontaneous remission, and may alternate from one side to the other. The aetiology is unknown and in the few cases, in which the pathology has been studied the lesion involving the cavernous sinus has been reported as nonspecific granulomatous tissue.? As in ophthalmoplegic migraine, carotid angiography in the Tolosa-Hunt syndrome has shown arterial narrowing in the cavernous sinus, but in addition the superior orbital veins have also been occluded. ${ }^{8}$ In our own experience computerised axial tomography of the orbit in the Tolosa-Hunt syndrome has shown areas of increased density in the apex of the orbit which also enhanced with contrast media, and it is of interest that similar changes have been reported in orbital pseudotumour. ${ }^{9}$ Hallpike has suggested that Tolosa-Hunt syndrome and orbital pseudotumour have pathological features in common.

It seems unlikely that our patient has had the Tolosa-Hunt syndrome in view of the large number of recurrences, their relatively stereotyped and unilateral nature, and the absence of involvement of other structures in the superior orbital fissure and orbital apex. The patient was not given corticosteroids at any stage. Although a prompt remission of pain followed by recovery of the deficit is an essential diagnostic criterion for the Tolosa-Hunt syndrome, steroids might be effective in ophthalmoplegic migraine, since their value in migrainous neuralgia has recently been described.10

Aberrant regeneration of the oculomotor nerve implies that there has been interruption of the axons with subsequent regrowth which has been misdirected. The signs of aberrant regeneration of the oculomotor nerve were confirmed in this patient 
by the presence of ipsilateral eyelid retraction on downward gaze and constriction of the pupil on attempted adduction and depression of the affected eye. These 2 characteristics are the result of axons intended for the inferior rectus growing into the branch to the levator and those for the medial and inferior rectus being misdirected to the pupil. The third feature of a typical case was not seen, namely, the adduction of the affected eye on attempted elevation or depression due to misdirection of fibres from superior and inferior rectus into the medial rectus.

Walsh and Hoyt ${ }^{11}$ noted that they had not observed aberrant regeneration of the third nerve in ophthalmoplegic migraine but they were aware of a single case. The fact that it does occur implies in some instances partial disruption of the nerve fibres of the peripheral or extra-axial portion of the oculomotor nerve during attacks. However, one would expect a higher incidence of aberrant regeneration if the lesion in ophthalmoplegic migraine were due to compression by an oedematous intracavernous section of the internal carotid artery. In addition one might expect a much higher incidence of sixth nerve palsy in view of its closer anatomical relationship to the artery. The rarity of aberrant regeneration of the oculomotor nerve in ophthalmoplegic migraine is akin to its rarity in diabetic oculomotor palsies, of which Walsh ${ }^{12}$ reported only 4 cases. Unlike diabetes, in which pupillary involvement in oculomotor palsies is unusual, internal ophthalmoplegia is commonly seen in ophthalmoplegic migraine. The pathology of the diabetic lesion has been established by Dreyfus et al..$^{13}$ and Asbury et al., ${ }^{14}$ who demonstrated demyelination of the central axons of the nerve, which they concluded, on the basis of severe hyaline degeneration of the intraneural arterioles, was ischaemic in origin. The frequent sparing of the pupil is attributed to the peripheral position of the parasympathetic fibres in the superior aspect of the oculomotor nerve. ${ }^{15}$

The oculomotor nerve receives its blood supply in the cavernous sinus mainly from branches of the tentorial artery, which is one of 3 divisions of the meningo-hypophyseal trunk, the principal branch of the intracavernous carotid artery. ${ }^{16}$ Asbury et al. ${ }^{14}$ also found that the nerve was supplied in its anterior portion by recurrent branches from the ophthalmic artery and in its posterior intracavernous course from the circle of Willis by small vessels arising from the posterior communicating and posterior cerebral arteries.

As an alternative explanation to direct compres- sion of the oculomotor nerve by an oedematous intracavernous carotid artery in ophthalmoplegic migraine Walsh and O'Doherty ${ }^{3}$ also suggested interference with the vasa nervorum of the nerve by the oedema. Walsh and Hoyt ${ }^{11}$ extended this concept to include occlusion of the ostia of the small arterial branches of the intracavernous carotid artery. The fact that aberrant regeneration of the oculomotor nerve does occasionally occur in migraine confirms that the lesion is in the peripheral portion of the nerve, and it is postulated that its relative rarity makes direct compression with resultant mechanical distortion by the internal carotid artery unlikely. Further it seems likely that the lesion is primarily ischaemic and due to occlusion of the numerous small vessels which supply the nerve in the region of the cavernous sinus, either by direct spasm or by oedema closing their ostia in the internal carotid artery.

\section{References}

${ }^{1}$ Charcot JM, cited by Rea RL. Neuro-ophthalmology. St Louis: Mosby, 1938.

${ }^{2}$ Harrington DO, Flocks M. Ophthalmoplegic migraine. Arch Ophthalmol 1953; 49: 643-55.

${ }^{3}$ Walsh JP, O'Doherty DS. A possible explanation of the mechanism of ophthalmoplegic migraine. Neurology 1960; 10: $1079-84$.

${ }^{4}$ Miller NR. Solitary oculomotor nerve palsy in childhood. Am J Ophthalmol 1977; 83: 106-11.

${ }^{5}$ Schatz NJ, Savino PJ, Corbett JJ. Primary aberrant oculomotor regeneration. Arch Neurol 1977; 34: 29-32.

${ }^{6}$ Hunt WE. Tolosa-Hunt syndrome, one cause of painful ophthalmoplegia. J Neurosurg 1976; 44: 544-9.

${ }^{7}$ Schatz NJ, Farmer P. Tolosa-Hunt syndrome: the pathology of painful ophthalmoplegia. Neuro-ophthalmology. St. Louis: Mosby, 1972: 6: 102-12.

${ }^{8}$ Hallpike JF. Superior orbital fissure syndrome: some clinical and radiological observations. J Neurol Neurosurg Psychiat 1973; 36: 486-90.

${ }^{9}$ Enzmann D, Donaldson SS, Marshall WH, Kriss JP. Computed tomography in orbital pseudotumour. Radiology 1976; 120: 597-601.

${ }^{10} \mathrm{James} \mathrm{JL}$. The treatment of cluster headaches with prednisone. Dis Nerv Sys 1975; 36: 375-6.

${ }^{11}$ Walsh FB, Hoyt WF. Clinical Neuro-ophthalmology, 3rd ed. Baltimore: Williams and Wilkins, 1969: 2.

${ }^{12}$ Walsh FB. Third nerve regeneration clinical evaluation. Br J Ophthalmol 1957; 41 : 577-98.

${ }^{13}$ Dreyfus PM, Hakim, Adams RD. Diabetic ophthalmoplegia: report of a case with post-mortem study and comments on vascular supply of human oculomotor nerve. Arch Neurol Psychiat 1957; 77: 337-49.

${ }^{14}$ Asbury AK, Aldredge H, Hershberg R, Fisher CM. Oculomotor palsy in diabetes mellitus: a clinico-pathological study. Brain 1970; 93: 555-66.

${ }^{15}$ Sunderland S, Hughes ESR. The pupillo-constrictor pathway and the nerve to the ocular muscles in men. Brain 1946; 69: 301-9.

${ }^{16}$ Parkinson D. Collateral circulation of cavernous carotid artery: anatomy. Can J Surg 1964; 7: 251-68. 